\title{
CATEGORICAL CONSTRUCTIONS FOR HOPF ALGEBRAS
}

\author{
A.L. AGORE \\ Dedicated to the memory of Professor Liliana Pavel
}

\begin{abstract}
We prove that both, the embedding of the category of Hopf algebras into that of bialgebras and the forgetful functor from the category of Hopf algebras to the category of algebras, have right adjoints; in other words: every bialgebra has a Hopf coreflection and on every algebra there exists a cofree Hopf algebra. In this way we give an affirmative answer to a forty years old problem posed by Sweedler. On the route the coequalizers and the coproducts in the category of Hopf algebras are explicitly described.
\end{abstract}

\section{INTRODUCTION}

Hopf algebras appeared naturally in the study of Lie groups cohomology. The survey paper 2] covers the beginnings of Hopf algebras and the roles played by H. Hopf, P. Cartier, A. Borel, J. Milnor, J. Moore, B. Konstant and M. Sweedler in the development of this theory. Hopf algebras became a fervid field of study especially after the appearance of the monograph [11]. In the present paper we bring new contributions to the study of the category of Hopf algebras.

We turn our attention to the fundamental book of Sweedler: in [11, p. 135] are stated, without any proofs, the following problems concerning Hopf algebras: given a coalgebra $C$ there exists a free Hopf algebra on $C$ (i.e. the forgetful functor from the category of Hopf algebras to the category of coalgebras has a left adjoint) and a free commutative Hopf algebra on $C$. The problem has turned out to be quite difficult: several years passed until Takeuchi, in [12, Sections $\S 1$ and $\S 11]$, answered affirmatively to both statements. His proof relies on an ingenious and laborious construction. Moreover, in [11, p. 135] Sweedler also states, again without any proofs, the dual of the above problem: given an algebra $A$ there exists a cofree Hopf algebra on $A$ (that is the forgetful functor from the category of Hopf algebras to the category of algebras has a right adjoint) and a cofree cocommutative Hopf algebra on $A$. Concerning this problem, recently H.-E. Porst [8, Corollary 4.1.4] proved that the existence of a cofree Hopf algebra on every algebra implies the existence of a cofree cocommutative Hopf algebra on every algebra. In the present paper we prove Sweedler's statement concerning the existence of a cofree Hopf algebra on every algebra.

2000 Mathematics Subject Classification. 18A30, 18A40.

Key words and phrases. bialgebra, Hopf algebra, (co)product, (co)limit, (co)complete, (co)refective.

The author acknowledges partial support from CNCSIS grant 24/28.09.07 of PN II "Groups, quantum groups, corings and representation theory". 
The paper is structured as follows. In Section 1 we introduce the notations and recall, without proofs, some well known results pertaining to category theory that will be intensively used throughout the paper. In Section 2 we give an explicit description of coequalizers and coproducts in the category $k$-HopfAlg of Hopf algebras. Using the aforementioned constructions we prove, using the Special Adjoint Functor Theorem, Sweedler's statement concerning the existence of a cofree Hopf algebra on every algebra (Theorem 2.5).

\section{Preliminaries}

Throughout this paper, $k$ will be a field. Unless specified otherwise, all vector spaces, algebras, coalgebras, bialgebras, tensor products and homomorphisms are over $k$. Our notation for the standard categories is as follows: ${ }_{k} \mathcal{M}$ ( $k$-vector spaces), $k$-Alg (associative unital $k$-algebras), $k$-BiAlg (bialgebras over $k$ ), $k$-HopfAlg (Hopf algebras over $k$ ). We refer to [11] for further details concerning Hopf algebras.

We use the standard notations for opposite and coopposite structures: $A^{\text {op }}$ denotes the opposite of the algebra $A$ and $C^{c o p}$ stands for the coopposite of the coalgebra $C$.

Let us recall briefly some well known results from category theory, refering the reader to 4] for more details. A category $\mathcal{C}$ is called (co)complete if all diagrams in $\mathcal{C}$ have (co)limits in $\mathcal{C}$. A category $\mathcal{C}$ is (co) complete if and only if $\mathcal{C}$ has (co)equalizers of all pairs of arrows and all (co)products [6, Theorem 6.10]. The category $\mathcal{C}$ is called locally small (or well-powered) if the subobjects of each $C \in \mathcal{C}$ can be indexed by a set. Dually, the category $\mathcal{C}$ is colocally small (or co-well-powered) if its dual is locally small. At some point we will also use, in passing, the notion of locally presentable category. More details regarding this type of categories can be found in [1]. All categories considered above are locally presentable. Thus, they are cocomplete by the definition of locally presentable categories and complete by [1, Remark 1.56]. For a more detailed discussion concerning the completeness and cocompleteness of the above categories we refer the reader to [8], [9] and [10]. A subcategory $\mathcal{D}$ of $\mathcal{C}$ is called (co)reflective in $\mathcal{C}$ when the inclusion functor $U: \mathcal{D} \rightarrow \mathcal{C}$ has a (right)left adjoint.

The following categorical result play a key role in showing that the category of Hopf algebras is a coreflective subcategory of the category of bialgebras:

Theorem 1.1. (The Special Adjoint Functor Theorem) If $\mathcal{C}$ is a complete and locally small category with a cogenerator, then a functor $G: \mathcal{C} \rightarrow \mathcal{D}$ has a left adjoint if and only if it is limit preserving. Dually, if $\mathcal{C}$ is a cocomplete and colocally small category with a generator, then a functor $G: \mathcal{C} \rightarrow \mathcal{D}$ has a right adjoint if and only if it is colimit preserving.

\section{Cofree Hopf algebras generated By algebras}

Recall that the forgetful functor from the category of groups to the category of monoids has a left adjoint, the so-called anvelopant group of a monoid, and a right adjoint, which assigns to each monoid the group of its invertible elements. Therefore, if we think of Hopf algebras as a natural generalization of groups, we may expect the same behavior in 
the case of the embedding functor $F: k$-HopfAlg $\rightarrow k$-BiAlg from the category of Hopf algebras to the category of bialgebras. It is well known that the above embedding functor $F$ has a left adjoint [5, Theorem 2.6.3]. We shall prove in this section that $F$ has also a right adjoint. This result together with that fact that there exist a cofree bialgebra on every algebra led us to the conclusion that the forgetful functor $F: k$-HopfAlg $\rightarrow k$-Alg has a right adjoint, as stated in [11. In order to prove our main result we need some preparations. We start by recalling, for a further use, the constructions of coequalizers and coproducts in the category $k$-BiAlg of bialgebras.

Let $\left(A, m_{A}, \eta_{A}, \Delta_{A}, \varepsilon_{A}\right),\left(B, m_{B}, \eta_{B}, \Delta_{B}, \varepsilon_{B}\right)$ be two bialgebras and $f, g: B \rightarrow A$ be two bialgebra maps. Consider $I$ the two-sided ideal generated by $\{f(b)-g(b) \mid b \in B\}$. By a simple computation it can be seen than $I$ is also a coideal. Then $(A / I, \pi)$ is the coequalizer of the morphisms $(f, g)$ in $k$-BiAlg, where $\pi: A \rightarrow A / I$ is the canonical projection. Indeed for all bialgebras $H$ and all bialgebra morphisms $h: A \rightarrow H$ such that $h \circ f=h \circ g$ we obtain $I \subseteq k e r h$, hence there exists an unique bialgebra map $h^{\prime}: A / I \rightarrow H$ such that $h^{\prime} \circ \pi=h$.

Remark 2.1. Note that if $A, B$ are two Hopf algebras and $f, g: B \rightarrow A$ are Hopf algebra maps then the ideal $I$ defined above is actually a Hopf ideal and $(A / I, \pi)$ is the coequalizer of the morphisms $(f, g)$ in $k$-HopfAlg, where $\pi: A \rightarrow A / I$ is the canonical projection.

Next, we recall from [5] the construction of the coproduct in the category $k$-BiAlg of bialgebras. Let $\left(A_{l}\right)_{l \in I}$ be a family of algebras, $\left(\bigoplus_{l \in I} A_{l},\left(j_{l}\right)_{l \in I}\right)$ be the coproduct in ${ }_{k} \mathcal{M}$ and $i: \bigoplus_{l \in I} A_{l} \rightarrow T\left(\bigoplus_{l \in I} A_{l}\right)$ be the canonical inclusion where $T\left(\bigoplus_{l \in I} A_{l}\right)$ is the tensor algebra of the vector space $\bigoplus_{l \in I} A_{l}$. Then $\left(\coprod_{l \in I} A_{l}:=T\left(\bigoplus_{l \in I} A_{l}\right) / L,\left(q_{l}\right)_{l \in I}\right)$ is the coproduct of the above family in $k$-Alg, where $L$ is the two sided ideal in $T\left(\bigoplus_{l \in I} A_{l}\right)$ generated by the set $J:=\left\{i \circ j_{l}\left(x_{l} y_{l}\right)-i\left(j_{l}\left(x_{l}\right)\right) i\left(j_{l}\left(y_{l}\right)\right), 1_{T}\left(\oplus A_{l}\right)-i \circ j_{l}\left(1_{A_{l}}\right) \mid x_{l}, y_{l} \in\right.$ $\left.A_{l}, l \in I\right\}, \nu: T\left(\bigoplus_{l \in I} A_{l}\right) \rightarrow T\left(\bigoplus_{l \in I} A_{l}\right) / L$ denotes the canonical projection and $q_{l}=$ $\nu \circ i \circ j_{l}$ for all $l \in I$. Furthermore, $\coprod_{l \in I} A_{l}$ is actually a bialgebra provided that $\left(A_{l}\right)_{l \in I}$ is a family of bialgebras. The comultiplication and the counit are given by the unique algebra maps such that the following diagrams commute:
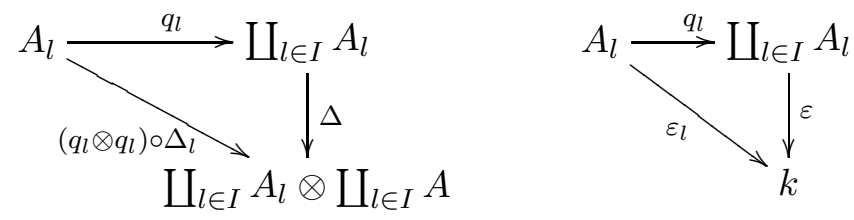

and $\coprod_{l \in I} A_{l}$ is the coproduct in $k$-BiAlg of the above family of bialgebras ([5, Corollary 2.6.2]).

Now let $\left(H_{l}, m_{l}, \eta_{l}, \Delta_{l}, \varepsilon_{l}, S_{l}\right)_{l \in I}$ be a family of Hopf algebras. Consider $\left(\left(H:=\coprod_{l \in I} H_{l}\right.\right.$, $\left.m, \eta, \Delta, \varepsilon),\left(q_{l}\right)_{l \in I}\right)$ the coproduct of the above family in the category $k$-BiAlg of bialgebras.

The universal property of the coproduct yields an unique bialgebra map $S: H \rightarrow H^{\text {opcop }}$ 
such that the following diagram commutes for all $l \in I$ :

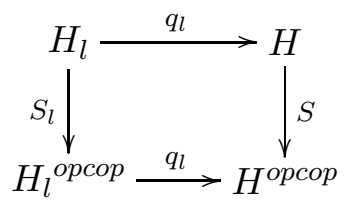

With the notations above we have the following result which provides a completely description of the coproducts in the category $k$-HopfAlg of Hopf algebras:

Theorem 2.2. Let $\left(H_{l}, m_{l}, \eta_{l}, \Delta_{l}, \varepsilon_{l}, S_{l}\right)_{l \in I}$ be a family of Hopf algebras. The Hopf algebra $\left(H:=\coprod_{l \in I} H_{l}, m, \eta, \Delta, \varepsilon, S\right)$ together with structure maps $\left(q_{l}\right)_{l \in I}$ is the coproduct in the category $k$-HopfAlg of the family $\left(H_{l}, m_{l}, \eta_{l}, \Delta_{l}, \varepsilon_{l}, S_{l}\right)_{l \in I}$ of Hopf algebras. In particular, the category $k$-HopfAlg is cocomplete.

Proof. We will first prove that $S$ is an antipode for the bialgebra $H$, i.e.

$$
m \circ(I d \otimes S) \circ \Delta=m \circ(S \otimes I d) \circ \Delta=\eta \circ \varepsilon
$$

Since $S: H \rightarrow H^{o p c o p}$ defined in (2) is a bialgebra map we only need to prove that (3) holds only on the generators of $H$ as an algebra. Indeed, let $h, k$ be generators in $H$ for which (3) holds. We obtain :

$$
(h k)_{(1)} S\left((h k)_{(2)}\right)=h_{(1)} k_{(1)} S\left(k_{(2)}\right) S\left(h_{(2)}\right)=\varepsilon(k) h_{(1)} S\left(h_{(2)}\right)=\varepsilon(h) \varepsilon(k) 1_{H}=\varepsilon(h k) 1_{H}
$$

It follows from here that (3) also holds for $k h$ and thus it holds for all elements in $H$. Now having in mind that $H:=T\left(\bigoplus_{l \in I} H_{l}\right) / L$ we only need to prove (3) for the elements $\widehat{x} \in H$ with $x \in \bigoplus_{l \in I} H_{l}$, whereas the tensor algebra $T\left(\bigoplus_{l \in I} H_{l}\right)$ is the free algebra on $\bigoplus_{l \in I} H_{l}$. Moreover, since $\bigoplus_{l \in I} H_{l}=\left\{x \in \prod_{l \in I} H_{l} \mid \operatorname{supp}(x)<\infty\right\}$ it is enough to show that (3) holds for all $x_{l} \in H_{l}, l \in I$. We then have:

$$
\begin{aligned}
& m \circ(I d \otimes S) \circ \Delta\left(\widehat{x}_{l}\right) \quad=\quad m \circ(I d \otimes S) \circ \Delta \circ q_{l}\left(x_{l}\right) \\
& \stackrel{11}{\underline{1}} \quad m \circ(I d \otimes S) \circ\left(q_{l} \otimes q_{l}\right) \circ \Delta_{l}\left(x_{l}\right) \\
& =\quad m \circ\left(q_{l} \otimes\left(S \circ q_{l}\right)\right) \circ \Delta_{l}\left(x_{l}\right) \\
& \stackrel{(2)}{=} \quad m \circ\left(q_{l} \otimes\left(q_{l} \circ S_{l}\right)\right) \circ \Delta_{l}\left(x_{l}\right) \\
& =\quad m \circ\left(q_{l} \otimes q_{l}\right) \circ\left(I d \circ S_{l}\right) \circ \Delta_{l}\left(x_{l}\right) \\
& q_{l}-\text { algebra map } \quad q_{l} \circ m_{l} \circ\left(I d \circ S_{l}\right) \circ \Delta_{l}\left(x_{l}\right) \\
& =\quad q_{l} \circ \eta_{l} \circ \varepsilon_{l}\left(x_{l}\right) \\
& q_{l}-\text { algebra map } \quad \eta \circ \varepsilon_{l}\left(x_{l}\right) \\
& q_{l}-\text { coalgebra map } \quad \eta \circ \varepsilon \circ q_{l}\left(x_{l}\right) \\
& =\quad \eta \circ \varepsilon\left(\widehat{x}_{l}\right)
\end{aligned}
$$

Hence $m \circ(I d \otimes S) \circ \Delta=\eta \circ \varepsilon$. In the same way it can be proved that $m \circ(S \otimes I d) \circ \Delta=\eta \circ \varepsilon$. Thus $S$ is an antipode for $H$, as desired. 
Now since $k$-HopfAlg is a full subcategory of the category $k$-BiAlg it follows that $((H:=$ $\left.\left.\coprod_{l \in I} H_{l}, m, \eta, \Delta, \varepsilon\right),\left(q_{l}\right)_{l \in I}\right)$ is also the coproduct of the family $\left(H_{l}, m_{l}, \eta_{l}, \Delta_{l}, \varepsilon_{l}, S_{l}\right)_{l \in I}$ of Hopf algebras in the category $k$-HopfAlg.

We need the following well known result:

Theorem 2.3. ([11, page 134]) The forgetful functor $F: k-B i A l g \rightarrow k-A l g$ has a right adjoint, i.e. there exists a cofree bialgebra on every algebra.

Our main results now follow:

Theorem 2.4. The embedding functor $F: k-H o p f A l g \rightarrow k-B i A l g$ has a right adjoint, i.e. the category of Hopf algebras is a coreflective subcategory of the category of bialgebras.

Proof. We apply the Special Adjoint Functor Theorem (Theorem 1.11): since $k$-HopfAlg is a locally presentable category by [8, 4.3.1 and 4.1.3] (although epimorphisms of Hopf algebras are not necessarily surjective maps [3]), this category in particular has a generator and is cocomplete and is colocally small. By Theorem 2.2 the result follows.

Theorem 2.5. The forgetful functor $F: k-H o p f A l g \rightarrow k-A l g$ has a right adjoint, i.e. there exists a cofree Hopf algebra on every algebra.

Proof. It follows from Theorem 2.3 and Theorem 2.4 by composing the right adjoint functors.

We proved, using the Special Adjoint Functor Theorem, the existence of a cofree Hopf algebra on every bialgebra without indicating explicitly his construction. The following natural problem arises:

Problem: Give an explicit construction of the cofree Hopf algebra on an bialgebra (resp. algebra).

We expect that the right adjoint of the embedding functor from the category of Hopf algebras to the category of bialgebras to assign to every bialgebra $B$ his "biggest" subbialgebra $H$ that has an antipode.

\section{ACKNOWLEDGEMENTS}

The author wishes to thank Professor Gigel Militaru, who suggested the problem studied here, for his great support and for the useful comments from which this manuscript has benefitted, as well as the referee for valuable suggestions and for indicating the papers [9] and [10]. 


\section{REFERENCES}

[1] J. Adámek, J. Rosický, Locally Presentable and Accessible Categories, Cambridge University Press, 1994

[2] N. Andruskiewitsch, W. F. Santos, The beginnings of the theory of Hopf algebras, to appear in Acta Appl. Math.

[3] A. Chirvasitu, On epimorphisms and monomorphisms of Hopf algebras, to appear in J. Algebra

[4] Mac Lane, S., Categories for the working mathematician, GTM 5, Springer, New York (1998)

[5] B. Pareigis, Lectures on quantum groups and noncommutative geometry. Available at: http://www.mathematik.uni-muenchen.de/ pareigis/Vorlesungen/02SS/QGandNCG.pdf

[6] B. Pareigis, Advanced Algebra. Available at: http://www.mathematik.uni-muenchen.de/ pareigis/Vorlesungen/01WS/advalg.pdf

[7] B. Pareigis, M.E. Sweedler, On generators and cogenerators, Manuscripta Math., 2 (1970), 49-66

[8] H.-E. Porst, Universal constructions for Hopf algebras, J. Pure Appl. Algebra, 212 (2008), 2547-2554

[9] H.-E. Porst, Fundamental constructions for coalgebras, corings and comodules, Appl. Categor. Struct., 16 (2008), 223-238

[10] H.-E. Porst, On corings and comodules, Arch. Math. (Brno), 42 (2006), 419-425

[11] M.E. Sweedler, Hopf Algebras, Benjamin New York, 1969

[12] M. Takeuchi, Free Hopf algebras generated by coalgebras, J. Math. Soc. Japan 23 (1971), 561-582

Department of Mathematics, Academy of Economic Studies, Piata Romana 6, RO-010374 Bucharest 1, Romania

E-mail address: ana.agore@fmi.unibuc.ro 